\title{
Unilateral Femoral Neck Fracture after Epileptic Seizure in Young Patient: A Case Report
}

\author{
J. C. Niyondiko ${ }^{*}$, P. Hakizimana1, P. Barasukana ${ }^{2}$, S. Manirakiza ${ }^{3}$, G. Ndayizeye ${ }^{1}$, \\ C. P. Baramburiye ${ }^{1}$, S. Nimubona ${ }^{1}$
}

\begin{abstract}
${ }^{1}$ Service de Chirurgie Orthopédique et Traumatologie, Centre Hospitalo-Universitaire de Kamenge, Bujumbura, Burundi ${ }^{2}$ Service de Neurologie, Centre Hospitalo-Universitaire de Kamenge, Bujumbura, Burundi ${ }^{3}$ Service de Radiologie, Centre Hospitalo-Universitaire de Kamenge, Bujumbura, Burundi Email: *niyjcc@yahoo.fr
\end{abstract}

How to cite this paper: Niyondiko, J.C., Hakizimana, P., Barasukana, P., Manirakiza, S., Ndayizeye, G., Baramburiye, C.P. and Nimubona, S. (2020) Unilateral Femoral Neck Fracture after Epileptic Seizure in Young Patient: A Case Report. Open Journal of Orthopedics, 10, 166-171.

https://doi.org/10.4236/ojo.2020.108019

Received: May 4, 2020

Accepted: August 4, 2020

Published: August 7, 2020

Copyright $\odot 2020$ by author(s) and Scientific Research Publishing Inc. This work is licensed under the Creative Commons Attribution International License (CC BY 4.0).

http://creativecommons.org/licenses/by/4.0/

(c) (i) Open Access

\begin{abstract}
Femoral neck fracture occurring after an epileptic seizure is a rare and under-diagnosed injury. The majority of the reported cases in literature are old patients with osteoporosis. Younger patients present several risk factors of osteopenia and the treatment remains controversial. We present an outcome of a 23 years old patient with unilateral femoral neck fracture occurring during an epileptic seizure and we discuss the associated multiple risk factors of osteopenia and osteonecrosis of the hip. The patient was brought to the emergency department of Teaching Hospital of Kamenge (CHUK) complaining of pain in his left hip that had been progressing for one month after an epileptic seizure. There is a history of HIV infection since birth and epileptic seizures with ongoing treatments for both diseases. Despite the high risk of avascular necrosis, the treatment choice has been influenced by the patient's age and a conservative surgery by internal fixation with Dynamic Hip Screw has been made. Unfortunately, this treatment early resulted in osteonecrosis of the hip since HIV infection itself and the highly active anti-retroviral therapy increase its risk.
\end{abstract}

\section{Keywords}

Femoral Neck Fracture, Epileptic Seizure, Osteopenia, Internal Fixation

\section{Introduction}

Epileptic seizures of grand mal type can be associated with fractures and/or dislocations of upper extremities of the limbs. These lesions are rare and under- 
diagnosed. They are consecutive to muscle contractions during generalized tonic-clonic seizures [1]. Literature review suggests that the risk of fracture is two to six times higher in epileptic patients because of repeated seizures and osteopenia [2].

Femoral neck fractures occurring simultaneously during an epileptic seizure have also been reported in the literature [1] [3]. They occur in elderly and osteoporotic patients with a mean age of 74.3 years and the treatment of choice is a hip replacement [2] [3] [4]. It can also occur in young patients with a high risk of osteopenia. Risk factors such as metastatic bone tumor, Paget's disease, hyperparathyroidism, anti-epileptic drugs, corticosteroids, HIV infection, antiretroviral therapy and alcohol abuse must be investigated [2]. In such cases, with younger patients, the treatment will be conservative in order to preserve the femoral head. The gold standard therapy is an internal fixation using Dynamic Hip screw (DHS) under C-arm control. However, there is a risk of avascular necrosis of the femoral head that can require a hip replacement especially when the risk factors mentioned above are found.

After obtaining the patient's consent, we report the follow up of a young patient with a unilateral femoral neck fracture that occurred after an epileptic seizure. We discuss the multiple risk factors of osteopenia and femoral necrosis.

\section{Case}

A 23 years old men was admitted to surgical emergency department of Kamenge Teaching hospital. He complained of a left hip pain and disability that occurred one month after a epileptic seizure. He is HIV positive since birth and has been treated with Duovir-N. Since the age of 2 years. The patient also reported a history of epileptic seizures treated with Dépakine, but he reports that this medication has been interrupted for several years.

The patient consulted a physician at a nearby medical center who prescribed a Nonsteroidal anti-inflammatory drug.

On admission, he was unable to walk. No history of trauma was reported. The left lower limb was shortened, in external rotation and adduction. The palpation aroused severe pain. The neurological and vascular examination was normal. A pelvic and left hip anteroposterior X-ray view was sufficient to confirm the diagnosis and revealed a Garden 3 femoral neck fracture (Figure 1).

An anti-epileptic treatment based on leptogin $200 \mathrm{mg}$ has been introduced.

Considering his age and despite the high risk of avascular necrosis of femoral head, an internal fixation using a Dynamic Hip Screw (DHS) was the preferred therapy; the objective being to obtain compression of the cervical fragment by restoring at least the cervico-obturator hanger and a cervico-diaphyseal angle within the normal range.

The early follow up was normal and the immediate post-operative radiological $\mathrm{x}$-ray showed that the reduction objectives had been achieved (Figure 2).

He underwent rehabilitation aiming to recover joints range of motion and left 
lower limb muscle strengthening. At three months of followup, the left hip was pain free. The control X-rays showed that fracture was healed without loss of reduction. Progressive weight bearing was then allowed.

At six months postoperative follow up, the patient was able to walk but with limping. X-ray showed a grade I avascular necrosis of the femoral head. After one year of follow up, he was still limping. The left hip was painless with the use of Diclofenac $100 \mathrm{mg}$ q.d. X-rays showed a grade IV avascular necrosis (Figure $3)$.

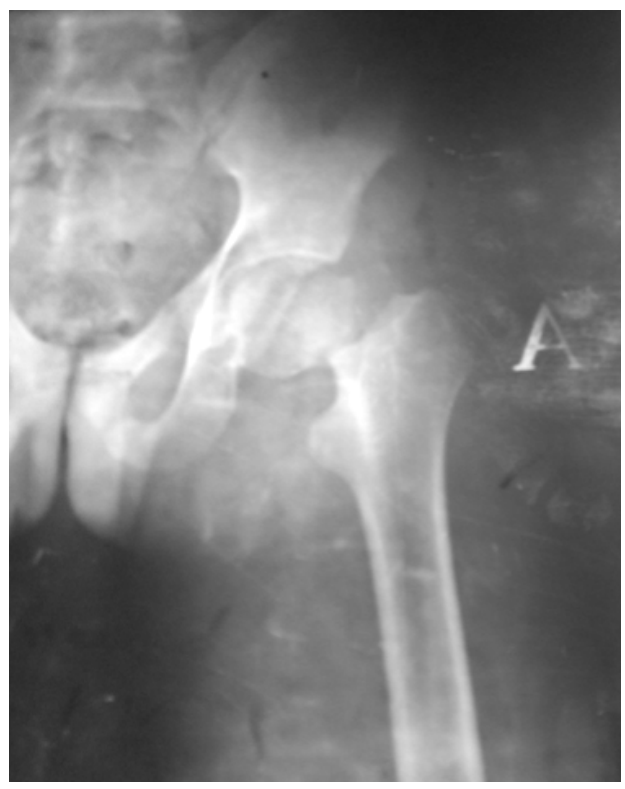

Figure 1. Preoperative anteroposterior x-ray: Left hip.

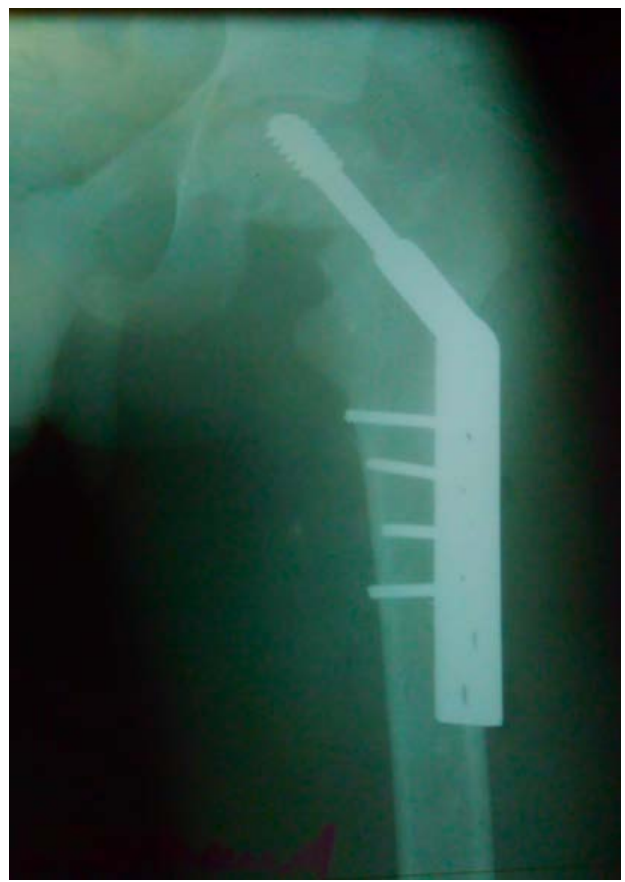

Figure 2. Postoperative anteroposterior x-ray: Left hip. 


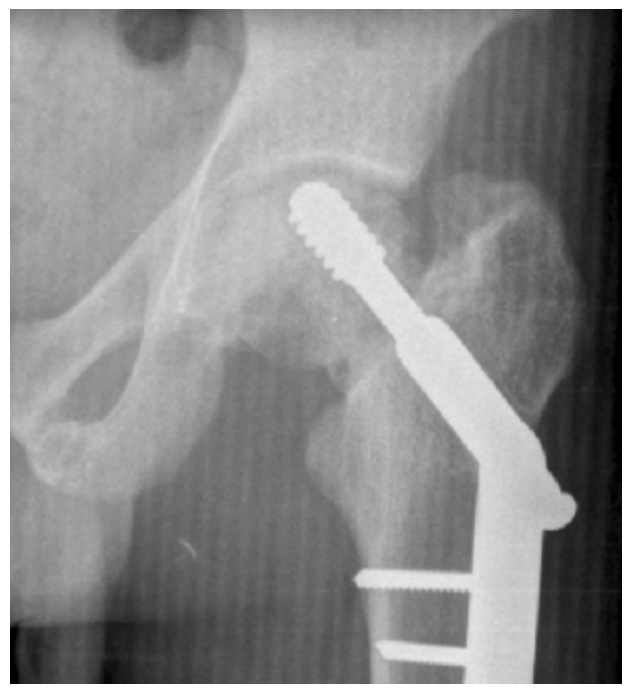

Figure 3. A 6 months anteroposterior x-ray: left hip.

\section{Discussion}

Rare cases of unilateral or bilateral femoral neck fractures following an epileptic seizure have been described in the literature since the 1970s [6]. They are all described in elderly patients (average age of 74.3 years) with osteoporosis and are linked to the violent contraction of the peri-articular muscles during the seizure [3] [4] [5].

Our patient was 23 years old but he had several risk factors of osteopenia. Therefore, HIV infection presents a bone toxicity via immune activation phenomena, which are harmful for bone metabolism. In fact, the activation of TCD4 + and CD8 + lymphocytes (HLA-DR + CD4 +, HLA-DR + CD8 +) is associated with lower bone mineral densities (BMD). In addition, immune restoration phenomena have an effect on bone metabolism. According to the cohorts analyzed, up to $50 \%$ of people infected with HIV are osteopenic and the prevalence of osteoporosis in this population can reach 15\% [7].

An increased incidence of osteopenia/osteoporosis has been associated with highly active anti-retroviral therapy (HAART) use which is based on combination of protease inhibitors (PIs) and nucleoside reverse transcriptase inhibitors (NRTIs). Our patient has been treated with Duovir-N for several years, a combination of two NRTIs; zidovudine and lamivudine. There are still many controversial issues regarding the pathogenesis and mechanisms leading to bone loss in HIV-infected patients on HAART, but the increased osteoclastogenesis induced by AZT is likely to play a critical role in the pathogenesis of osteopenia and osteoporosis [8].

Several studies have also pointed out that the risk of fracture in epilepsy patients is increased by 2 to 6 times. In fact, some anti-epileptics, by increasing bone turnover, reduce bone mineral density, which increases the risk of fracture [9].

Pack [10] recommends vitamin $\mathrm{D}$ and calcium supplementation as well as 
bone mineral density control in patients on long term anti-epileptic therapy, especially if they have other risk factors of osteopenia.

Despite the recent invention of types of hip prosthetic implants with a long lifespan and the high risk of osteonecrosis and osteoarthritis, we preferred an internal fixation with DHS that would allow him to live with "normal" hip and delay the hip arthroplasty. Nevertheless, our patient showed early signs of femoral head necrosis. Apart from the type of fracture and delayed treatment that can lead to avascular necrosis, HIV infected patients have an increased risk for osteonecrosis of the hip but it is not known weather it is attributable to HIV infection itself, HAART or other HIV-associated complications [11].

There is a discrepancy between the clinical and the radiological signs for our patient as he is able to walk without crutches with a painless hip. Total hip replacement(THR) will be decided considering the onset of pain and the quality of life.

\section{Conclusion}

Fractures of the femoral neck following an epileptic seizure are rare and underdiagnosed. They often occur in old patients with osteoporosis. In young patients, there are often a combination of risk factors of osteopenia. In our case, HIV infection, use of highly active anti-retroviral therapy and anti epileptic medication was the involved risk factors. In such a case, a conservative treatment early leads to avascular necrosis since HIV infection and HAART increase the risk of osteonecrosis of the hip.

\section{Conflicts of Interest}

The authors declare no conflicts of interest regarding the publication of this paper.

\section{References}

[1] Rahman, M.M. and Awada, A. (2003) Bilateral Simultaneous Hip Fractures Secondary to an Epileptic Seizure. Saudi Medical Journal, 24, 1261-1263.

[2] Khurram, S., Lambros, A. and Philip Housden, P. (2011) Spontaneous Bilateral Neck of Femur Fractures and Shoulder Dislocation. BMJ Case Reports. https://doi.org/10.1136/bcr.05.2010.3034

[3] Gur, S., Yilmaz, H., Tuzuner, S. and Suleymanlar, G. (1999) Fractures Due to Hypocalcemic Convulsion. International Orthopaedics, 23, 308-309. https://doi.org/10.1007/s002640050377

[4] Ribocoba-Montero, R. and Salas-Puig, J. (1997) Simultaneous Bilateral Fracture of the Hip Following a Grand Mal Seizure. An Unusual Complication. Seizure, 6, 403406. https://doi.org/10.1016/S1059-1311(97)80040-4

[5] Marsh, J.P., Leiter, J.R.S. and MacDonald, P. (2010) Bilateral Femoral Neck Fractures Resulting from a Grand Mal Seizure in an Elderly Man with Down Syndrome. Orthopedic Reviews, 2, 301-302. https://doi.org/10.4081/or.2010.e10

[6] Hughes, C.A. and O'Briain, D.S. (2000) Sudden Death from Peivic Hemorrhage after Bilateral Central Fracture Dislocations of the Hip Due to an Epileptic Seizure. 
The American Journal of Forensic Medicine and Pathology, 21, 380-384. https://doi.org/10.1097/00000433-200012000-00017

[7] Gazzola, L., Bellistri, G.M., Tincati, C., et al. (2013) Association between Peripheral T-Lymphocyte Activation and Impaired Bone Mineral Density in HIV-Infected Patients. Journal of Translational Medicine, 11, 51. https://doi.org/10.1186/1479-5876-11-51

[8] George, P., Zhen, Y., Scott, W.B. and Jay, M.M. (2006) Pathogenisis of Osteopenia/Osteoporosis Induced by Highly Active Anti-Retroviral Therapy for AIDS. Annals of the New York Academy of Sciences, 1068, 297-308. https://doi.org/10.1196/annals.1346.057

[9] Pack, A.M. and Morrel, M.J. (2004) Epilepsy and Bone Health in Adults. Epilepsy \& Behavior, 5, 24-29. https://doi.org/10.1016/j.yebeh.2003.11.029

[10] Pack, A. (2008) Bone Health in People with Epilepsy: Is It Impaired and What Are the Risk Factors? Seizure, 17, 181-186. https://doi.org/10.1016/j.seizure.2007.11.020

[11] Allison, G.T., et al. (2003) Osteonecrosis in HIV Disease: Epidemiology, Ethiology, and Clinical Management. AIDS, 17, 1-9.

https://doi.org/10.1097/01.aids.0000042940.55529.93 